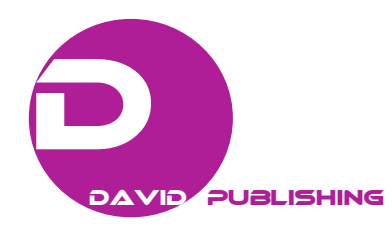

\title{
Multinomial Logistic Regression Model for Predicting Driver's Drowsiness Using Only Behavioral Measures
}

\author{
Atsuo Murata and Kensuke Naitoh \\ Department of Intelligent Mechanical Systems, Okayama University, Okayama 700-8530, Japan
}

\begin{abstract}
The aim of this study was to explore the effectiveness of behavioral evaluation measures for predicting drivers' subjective drowsiness. Behavioral measures included neck bending angle, back pressure, foot pressure, COP (center of pressure) movement on sitting surface and tracking error in driving simulator task. Drowsy states were predicted by means of the multinomial logistic regression model where behavioral measures and subjective evaluation of drowsiness corresponded to independent variables and a dependent variable, respectively. First, we compared the effectiveness of two methods (correlation coefficient-based method and odds ratio-based method) for determining the order of entering behavioral measures into the prediction model. It was found that the prediction accuracy did not differ between both methods. Second, the prediction accuracy was compared among the numbers of behavioral measures. The prediction accuracy did not differ among four, five and six behavioral measures and it was concluded that entering at least four behavioral measures into the prediction model is enough to achieve higher prediction accuracy. Third, the prediction accuracy was compared between the strongly drowsy and the weakly drowsy groups. The prediction accuracy differed between the two groups and the proposed method was effective under the condition where drowsiness was induced to a larger extent.
\end{abstract}

Key words: Drowsy driving, traffic accident, physiological measures, behavioral measures, prediction accuracy, multinomial logistic regression, subjective drowsiness.

\section{Introduction}

From the viewpoint of automotive preventive safety, effective measures for monitoring drowsiness during driving, detecting a decrease of arousal level and warning drivers of the risk of causing a traffic accident must be established so as to prevent drivers from driving under drowsy state and causing a disastrous traffic accident. In order to realize the development of such a system, not only the gross tendency of reduced arousal level but also the more accurate prediction of the state when the drowsiness occurs is indispensable. It is not until such accurate method to predict the occurrence of risky and drowsy driving is established that we apply this prediction technique to the development of automotive preventive safety system which can support so that drivers can avoid unsafe and drowsy driving.

Corresponding author: Atsuo Murata, Ph.D., professor, research fields: cognitive engineering and automotive ergonomics. E-mail: murata@iims.sys.okayama-u.ac.jp.
A few studies used psychophysiological measures such as blink, saccade, EEG (electroencephalography) and ECG (electrocardiography) and made an attempt to assess drowsiness or fatigue [1-3]. These studies showed that psychophysiological measures were, to some extent, correlated with subjective rating on drowsiness or fatigue especially at the end of the field or laboratory experiment. Kecklund and Akersted [3] carried out a field study and showed that end-of-drive subjective sleepiness and the EEG alpha burst activity were significantly correlated with total work hours. No measures alone can be used reliably to assess sleepiness or drowsiness. Moreover, these studies did not make an attempt to predict but the drowsy state using these psychphysiological measures.

Murata and Hiramatsu [4] and Murata and Nishijima [5] objectively evaluated the drowsiness of participants under simulated driving environment using EEG or HRV (heat rate variability) measures. They succeeded in clarifying the decrease of 
EEG-MPF (mean power frequency) or the increase of RRV3 when the participant's arousal level is low. However, it was not possible for these studies to predict the drowsiness on the basis of the time series of EEG-MPF or RRV3.

Murata et al. [6] applied Bayesian theorem and proposed a method to evaluate the arousal level using EEG, heart rate variability and tracking error during the simulated driving task. However, this study did not make an attempt to predict the arousal level. Murata et al. [7] and Murata et al. [8] applied a logistic regression model to mainly physiological measures such as EEG, ECG or EOG (electrooculography) in order to predict the arousal level (the subjective rating on drowsiness) and attained a prediction accuracy of about $85 \%$.

Such equipments to measure EEG or HRV are too expensive to put these systems into practical use in automotives. The drowsiness prediction system that should be used in automotive cockpit must be less expensive and more convenient. As a more convenient measure for predicting the arousal level, we paid attention to the vertical and horizontal neck bending angles and the change of COP (center of pressure) of the sitting surface. Murata et al. [9], Murata et al. [10] and Murata et al. [11] used behavioral measures such as tracking error in simulated driving task, back and foot pressure and COP during sitting pressure measurement and demonstrated that behavioral measures are as effective as physiological measures such as EEG-MPF or RRV3.

As mentioned above, a larger part of studies on drowsiness evaluation or prediction pay attention to both physiological and behavioral measures. In order to prevent traffic accidents due to drowsy driving, a lot of attempts are made to detect the tendency of decreased arousal level using physiological measures such as ECG, EEG or EOG, or performance measures such as a tracking error. Generally, it has been demonstrated that such physiological measures respond sensitively to the change of arousal level.
Until now, there are no useful methods to predict drowsiness with high reliability. Methods using physiological measures are not practical due to expensive price of their measurement apparatus. From the practical viewpoint, only behavioral measures were used for the drowsiness prediction.

The aim of this study was to explore whether only behavioral measures can predict the drowsiness with high accuracy. The neck bending angle (horizontal and vertical), back pressure, foot pressure, COP movement on sitting surface and tracking error in driving simulator task were taken up as behavioral measures for predicting the subjective rating on drowsiness. We used the multinomial logistic regression to predict the subjective rating on drowsiness (dependent variable) by entering the behavioral measures into the prediction model. From a practical viewpoint, it is desirable to obtain high accuracy with fewer behavioral measures. In this study, the following three issues were discussed:

(1) proposal of multinomial logistic regression model that can predict the subjective drowsiness with high accuracy by using only behavioral measures;

(2) effectiveness of two methods (correlation coefficient-based method and odds ratio-based method) for determining the order of entering behavioral measures into the prediction model;

(3) determination of minimum number of behavioral measures that can predict the subjective drowsiness with high accuracy;

(4) comparison of prediction accuracy between strongly drowsy and weakly drowsy groups.

On the basis of the investigations above, some implications for the practicability of the proposed prediction model were given.

\section{Method}

\subsection{Participants}

Thirteen healthy male undergraduate students from 21 years to 24 years old took part in the experiment. The visual acuity of the participants was matched and 
more than 20/20. They had no orthopedic or neurological diseases. All participants provide the experimenter with informed consent on the participation to the experiment. They were required to stay up all night and visit the laboratory. In this way, we carried out an experiment so that the participants readily felt asleep or carried out an experimental task under a drowsy or low arousal state.

\subsection{Apparatus}

The outline of simulated driving system is shown in Fig.1. In Fig. 1a, the display of simulated driving task is shown. The steering wheel used for simulated driving task and switch location for evaluating subjective drowsiness every $1 \mathrm{~min}$ are shown in Fig. 1b. The detailed display of inside lane in the simulated driving task is shown in Fig. 2. The display consisted of three lanes, and the width of each lane corresponded to $3.6 \mathrm{~m}$. The measurement of neck bending angle (Fig. 3a) and the apparatus for measuring the pressure on the sitting surface are shown in Fig. 3. DKHs (goniometers) were attached to the back of the neck to measure the neck bending angle. A measurement system of sitting pressure distribution Conform-Light (Nitta) was placed on a driver's seat. Eight pressure sensors OctSense (Nitta) were attached to the shoes insole for measuring foot

pressure. The eight pressure sensors were attached to the backrest of the driving seat for measuring back pressure (Fig. 4).

\subsection{Task}

The participants sat on an automobile seat and were required to carry out a simulated driving task. The display of the driving simulator is depicted in Fig. 1. The participants were required to steer a steering wheel and keep their vehicle to the center line as much as they could. If the participant kept the distance between two cars to a moderate level, the following car was encompassed by a green rectangle. If the distance between two cars was too short or too long, the color of the encompassed rectangle changed to different color (red for short distance or blue for long distance between two cars).

The psychological rating included the following three categories: (1) arousal; (2) a little drowsy; (3) very drowsy. It is possible to use a more detailed category such as 7-point or 5-point category. The following advantages and disadvantages exist in such categorization. While the finer categorization enables us to conduct finer evaluation of drowsiness, the disadvantage of such categorization is that the exact evaluation according to finer categories is difficult and suffers from more frequent false (ambiguous) evaluation (a)

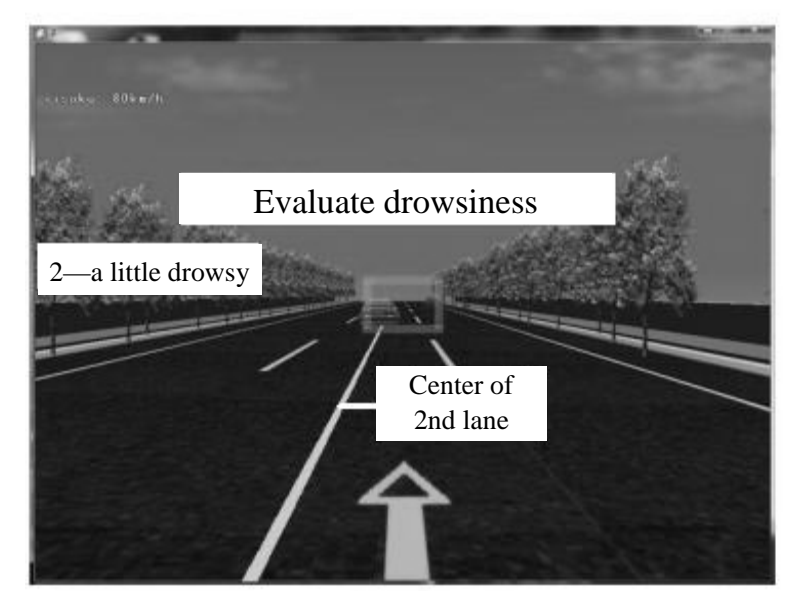

Fig. 1 Explanation of driving simulator and steering wheel used in the experiment: (a) display of driving simulator; (b) steering wheel used for simulated driving task.

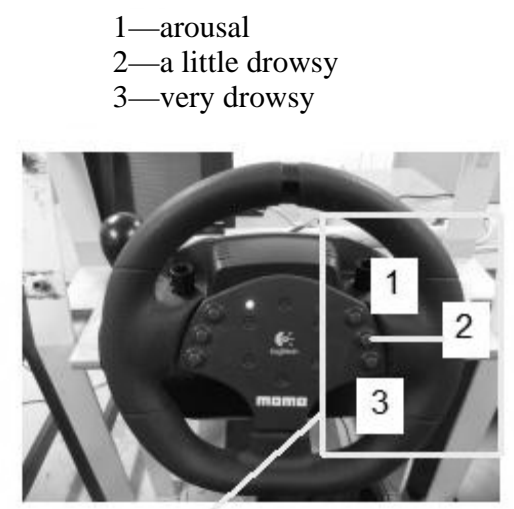

Button to be used for drowsiness evaluation

(b) 


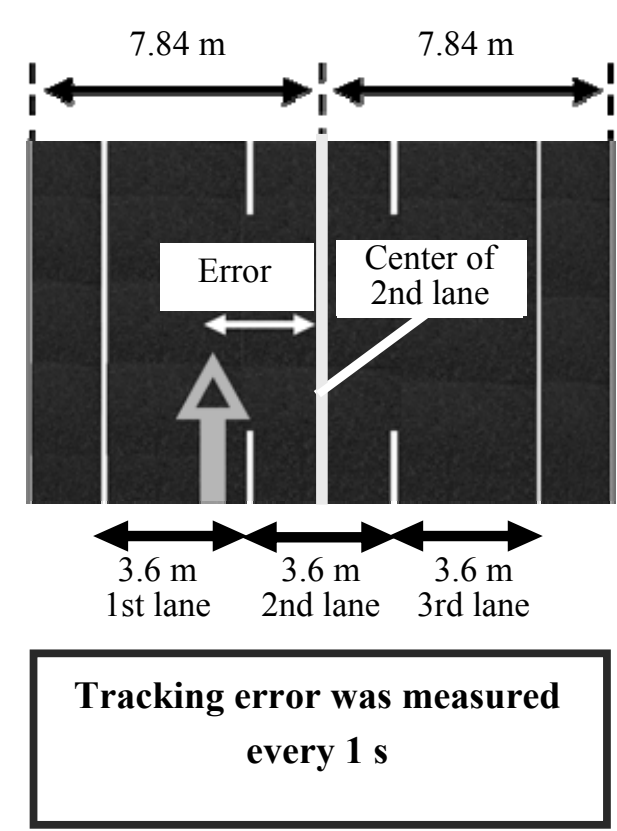

Fig. 2 Detailed display of inside lane in the simulated driving task.

at the boundary of two arbitrary categories. Moreover, it is pointed out that the more alternative the choice is, the more difficult the choice is [12]. In this study, the participant must report their subjective rating on drowsiness every $1 \mathrm{~min}$. The more alternatives force the participants to feel it difficult to evaluate their feeling, it is more possible that the subjective evaluation cannot be carried out properly. Therefore, we adopted three-point categorization of drowsiness. The participant was required to evaluate his drowsiness using the switches $1 \sim 3$ in Fig. 1 every 1 min.

\subsection{Procedure}

The psychological rating of drowsiness was checked every $1 \mathrm{~min}$. Behavioral measures included neck bending angle (horizontal and vertical), back pressure, foot pressure, COP movement on sitting surface and tracking error in driving simulator task. The neck bending angle was sampled with the sampling frequency of $10 \mathrm{~Hz}$. The foot pressure, the back pressure and COP on sitting surface were sampled with the sampling frequency of $50 \mathrm{~Hz}$. The tracking error (deviation of arrow in Figs. 1 or 2 from the center of 2nd lane) was measured every $1 \mathrm{~s}$.

Behavioral measures above were recorded while performing a simulated driving task under the low arousal condition. The duration of experimental task differed among the participants because the extent of induced drowsiness differed among the participants. Deliberating the degree of drowsiness of each participant, the experiment was continued for at most $90 \mathrm{~min}$. Applying these measures to the multinomial logistic regression models, the prediction accuracy of drowsiness was examined from a variety of conditions: (1) methods for determining the order of entering behavioral measures into the prediction model; (2) number of behavioral measures used for prediction with high accuracy; (3) comparison of prediction accuracy between strongly drowsy and weakly drowsy groups.

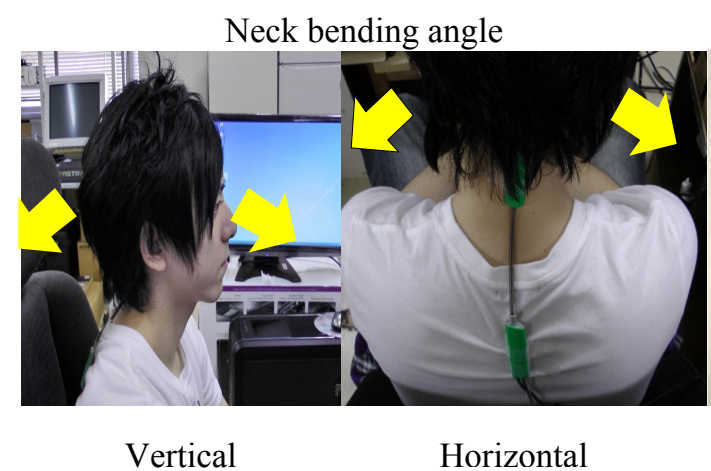

(a)

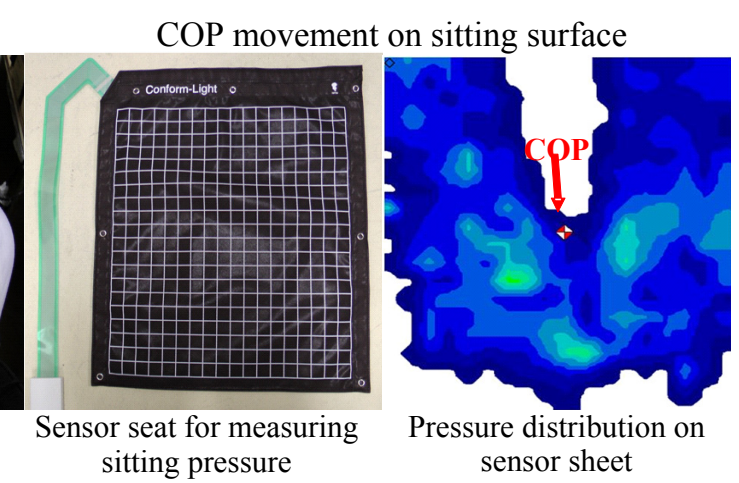

(b)

Fig. 3 Measurement of: (a) neck bending angle (DKH sampled every $1 \mathrm{~s}$ ); (b) pressure on sitting surface (sensor sheet for measuring sitting pressure (Nitta, Conform-Light), sampled every 0.02 s). 


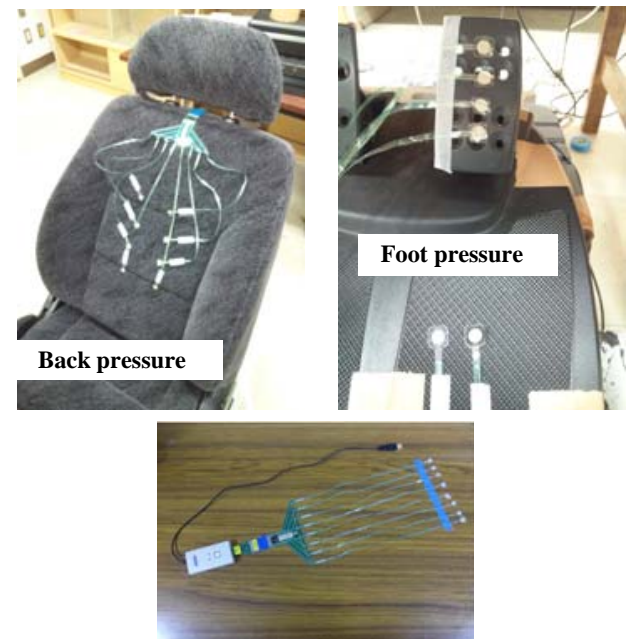

Fig. 4 Measurements of back pressure and foot pressure (Octsense (Nitta Corporation), sampled every $0.5 \mathrm{~s}$ ).

\subsection{Data Processing}

The procedure for predicting subjective drowsiness is summarized in Fig. 5. An attempt was made to predict subjective drowsiness by applying multinomial logistic regression to measured data. The following behavioral measures were included in measured data: tracking error in simulated driving task, neck bending angle (horizontal), neck bending angle (vertical), back pressure, foot pressure and movement of COP. The movement of COP was calculated and the difference of two consecutive values of COP was calculated. As COP was measured every $0.02 \mathrm{~s}$ (sampling frequency of $50 \mathrm{~Hz}$ ), there were 500 data of COP. The sum of this value for $10 \mathrm{~s}$ was obtained. The mean of this value was calculated as a representative of the movement of COP.

As the subjective rating on drowsiness was recorded every $1 \mathrm{~min}$, the mean values for $1 \mathrm{~min}$ were used as representatives of behavioral measures so that these behavioral measures can be entered into the multinomial logistic regression.

The following multinomial logistic regression was used to predict the subjective drowsiness expressed from 1 ("arousal") to 3 ("very drowsy"). The dependent variable was the subjective drowsiness and the independent variables corresponded to the behavioral measures mentioned above. The probability of each category $P(1$ : arousal $), P(2$ : a little drowsy) and $P(3$ : very drowsy) can be calculated using the following equations:

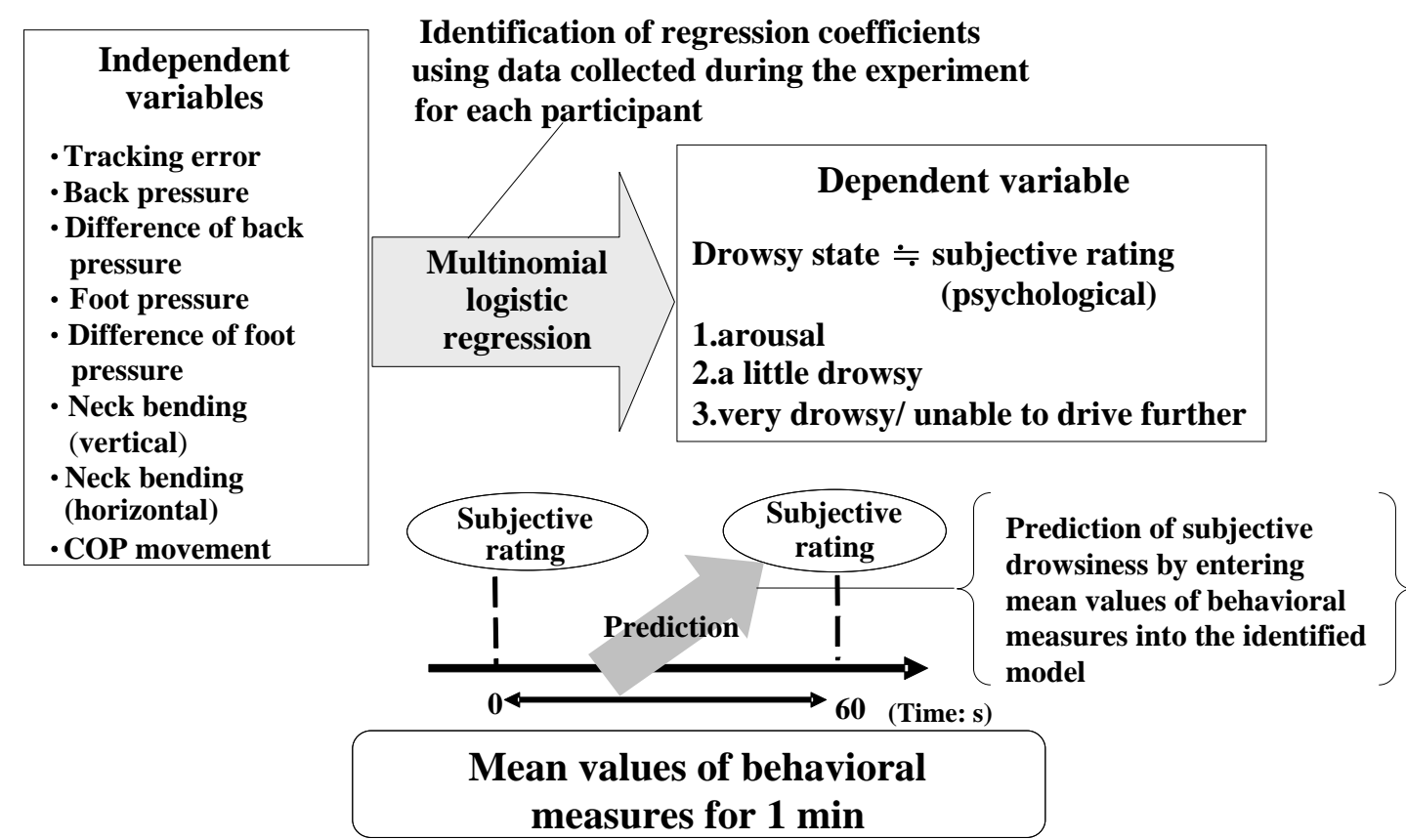

Fig. 5 Procedure of predicting subjective drowsiness by multinomial logistic regression. 


$$
\begin{aligned}
& P(2: \text { a little drowsy })= \\
& \frac{\exp \left(b_{0}+b_{1} x_{1}(2)+\cdots \cdots+b_{n} x_{n}(2)\right)}{1+\exp \left(b_{0}+b_{1} x_{1}(2)+\cdots \cdots+b_{n} x_{n}(2)\right)} \\
& P(3: \text { very drowsy })= \\
& \quad \frac{\exp \left(b_{0}+b_{1} x_{1}(3)+\cdots \cdots+b_{n} x_{n}(3)\right)}{1+\exp \left(b_{0}+b_{1} x_{1}(3)+\cdots \cdots+b_{n} x_{n}(3)\right)} \\
& P(1: \text { arousal })= \\
& 1-P(2: \text { a little drowsy })-P(3 \text { : very drowsy })
\end{aligned}
$$

where, $x_{1}, x_{2}, x_{3}, \ldots, x_{n}$ represent the value of behavioral measures and $n$ corresponds to the number of behavioral measures; $x_{1}(2), \ldots, x_{n}(2)$ show the values of each evaluation measure when the corresponding subjective evaluation is equal to 2 ; $x_{1}(3), \ldots, x_{n}(3)$ represent similar values when the subjective evaluation is equal to 3 . According to the calculated probability $P(1), P(2)$ and $P(3)$, the prediction of the subjective drowsiness was conducted. The category corresponding to the maximum value of $P(1), P(2)$ and $P(3)$ was estimated as the prediction of subjective drowsiness. If the value of $P(3)$ was maximum, the prediction of subjective drowsiness was regarded as 3 (very drowsy).

As mentioned above, the duration of experiment differed among the participants and ranged from 60 min to $90 \mathrm{~min}$, because the extent of induced drowsiness differed among the participants. The total number of data (subjective rating on drowsiness and behavioral measures) was between 60 and 90 . Applying the total number of data to the multinomial logistic regression above, the regression coefficients were obtained. As shown in Fig. 5, mean values of behavioral measures were entered into the identified multinomial logistic regression equation in order to obtain the predicted values of subjective drowsiness. The actual and the predicted values on subjective rating on drowsiness were used to obtain the prediction accuracy. If the predicted value coincides with the actual value, for example, 81 times out of 90 data, the prediction accuracy can be calculated as 0.9 $(=81 / 90)$.

\subsection{Determination of Order of Entering Behavioral Measures into the Prediction Model}

The order of entering behavioral measures into the prediction model was determined by either correlation coefficient-based method or odds ratio-based method. The tracking error was firstly and unconditionally entered into the logistic regression model. The correlation coefficients between the tracking error and the five behavioral measures (back pressure, COP movement, neck bending angle (vertical), neck bending angle (horizontal) and foot pressure) were calculated. The behavioral measures above were entered into the model in ascending order of correlation coefficient. In other words, the measure whose correlation coefficient with the tracking error was lower was preferentially entered into the multinomial logistic regression.

The determination of entry of behavioral measures into the prediction model by odds ratio-based method is explained. Denoting the value of a behavioral measure and the probability of behavioral value taking such a value as $x$ and $y$, respectively, the multinomial logistic regression model can be expressed as follows:

$$
y=\frac{\exp (a x+b)}{1+\exp (a x+b)}
$$

where, the parameters $a$ and $b$ denote logistic regression coefficients. The odds ratio of this model $X$ can be expressed as follows:

$$
X=\frac{y}{1-y}
$$

The more distant the odds ratio is from 1 , the stronger the behavioral measure is related to the variation of variable $y$. The odds ratios for the five behavioral measures above (back pressure, COP movement, neck bending angle (vertical), neck bending angle (horizontal) and foot pressure) were calculated. The behavioral measures above were 
entered into the model in descending order of odds ratio. In other words, the measure which has a higher odds ratio with the dependent variable (subjective drowsiness) was preferentially entered into the multinomial logistic regression model.

\section{Results}

In Fig. 6, the prediction accuracy is compared among behavioral measures for the multinomial logistic regression using a single measure as an independent variable. In Fig. 7, we showed the correlation coefficient between tracking error and five behavioral measures. The circled numbers 2 6 were attached according to the value of correlation coefficient. The circled numbers 2 and 6 correspond to the highest and the lowest correlation coefficient, respectively. Fig. 8 shows the comparison of prediction accuracy among the numbers of entered behavioral measures into the multinomial logistic regression model. The independent variables were entered into the model according to the circled number in Fig. 7.

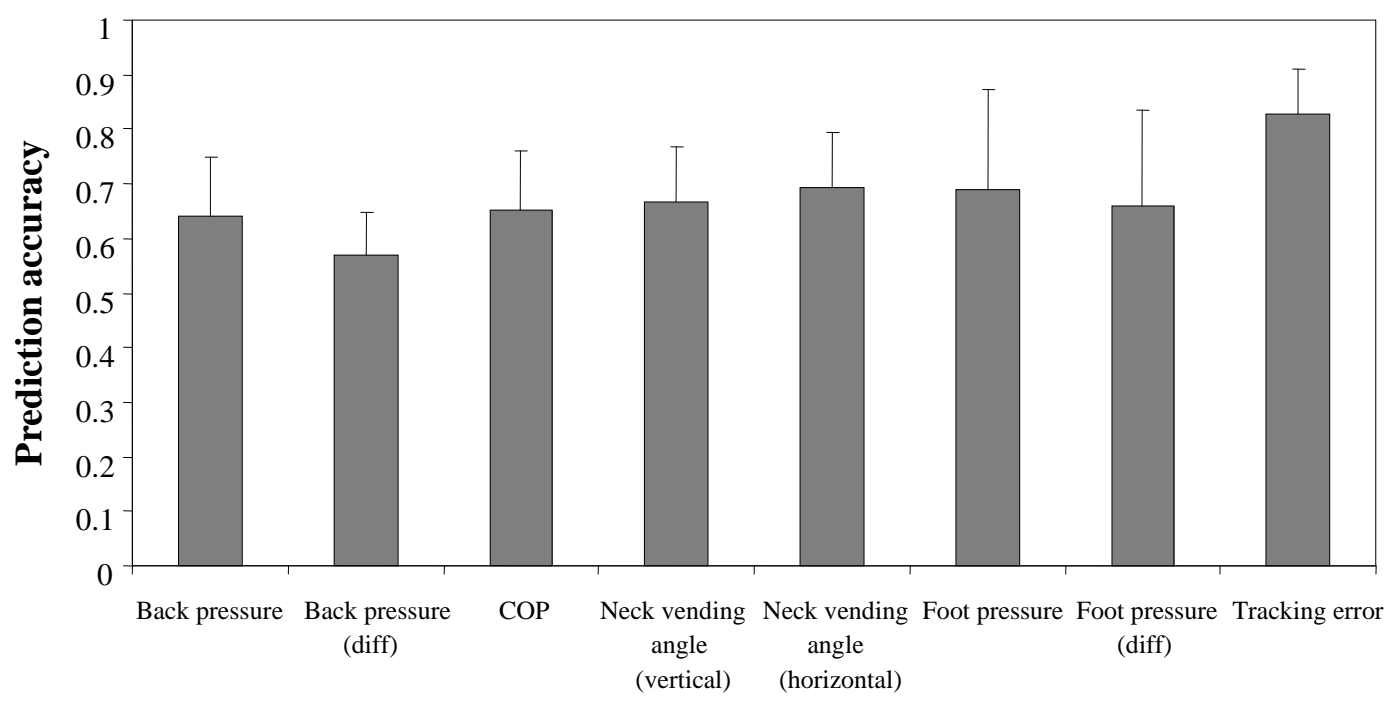

Fig. 6 Comparison of prediction accuracy of subjective drowsiness among behavioral measures (multinomial logistic regression using a single measure as an independent variable).

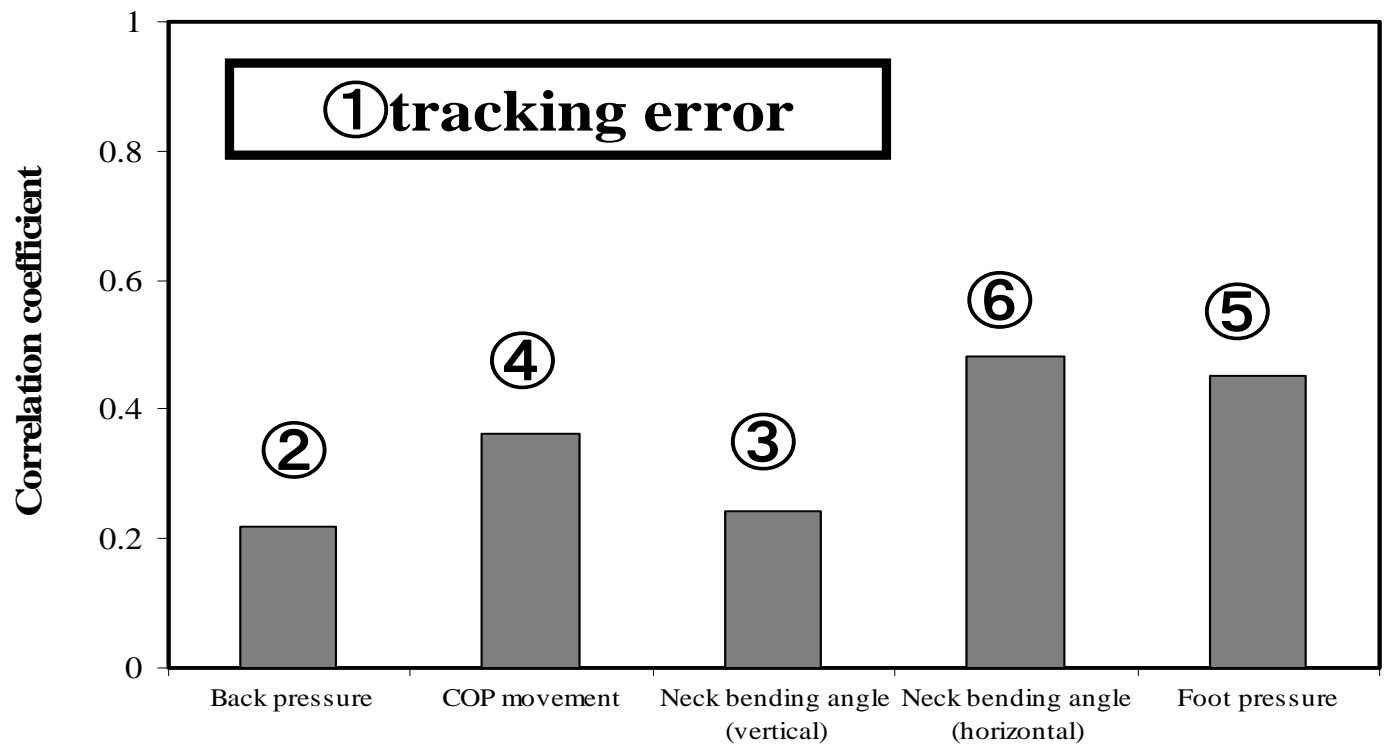

Fig. 7 Correlation coefficient between tracking error and five behavioral measures (back pressure, COP movement, neck bending angle (vertical), neck bending angle (horizontal) and foot pressure). 


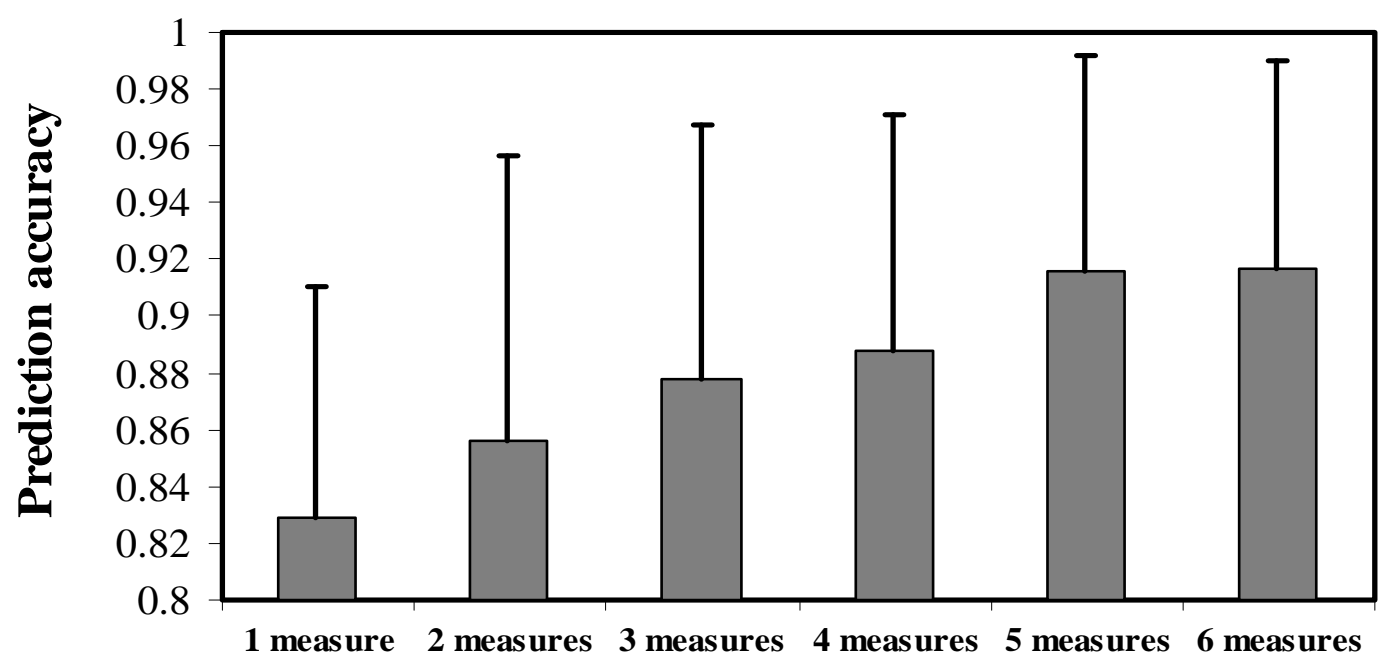

Fig. 8 Comparison of prediction accuracy among the numbers of behavioral measures entered into the multinomial logistic regression model. The independent variables were entered into the model according to the circled number in Fig. 7. For two measures, the circled numbers 1 (tracking error) and 2 (back pressure) were entered. For three measures, the circled numbers 1, 2 and 3 were entered. Alike, for six measures, the circled numbers 1 to 6 were entered.

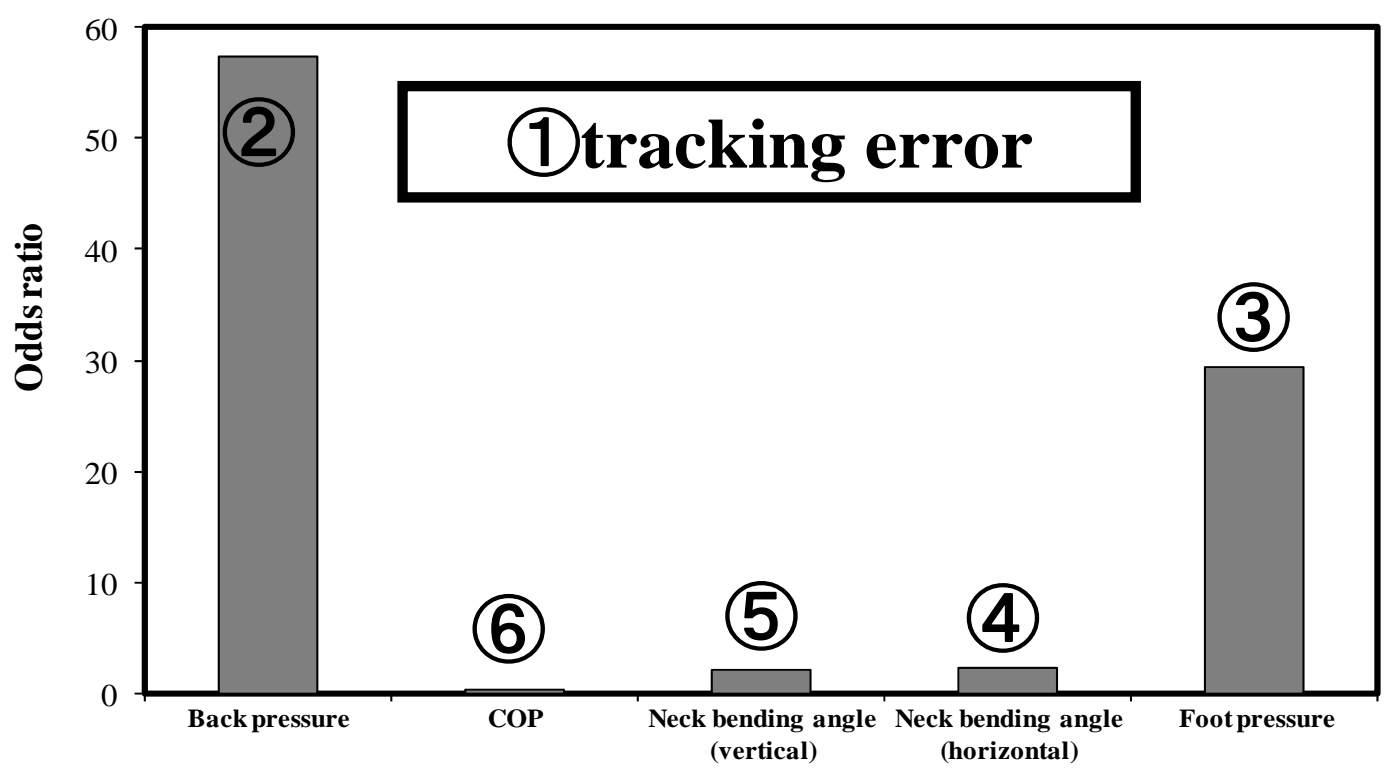

Fig. 9 Odds ratio of five behavioral measures (back pressure, COP movement, neck bending angle (vertical), neck bending angle (horizontal) and foot pressure).

Fig. 9 shows the odds ratio of five behavioral measures (back pressure, COP movement, neck bending angle (vertical), neck bending angle (horizontal) and foot pressure). Fig. 10 shows the prediction accuracy as a function of the numbers of entered measures into the multinomial logistic regression model. The independent variables were entered into the model according to the circled number in Fig. 9. For one measure, only the tracking error was entered into the prediction model. For two measures, the circled numbers 1 (tracking error) and 2 (back pressure) were entered. For three measures, the circled numbers 1, 2 and 3 (foot pressure) were entered. Alike, for six measures, the circled numbers 1 to 6 were entered.

\section{Discussion}

\subsection{Determination of Order of Entering Behavioral Measures into the Prediction Model}

As shown in Fig. 6, the prediction accuracy of the tracking error was by far higher than that of other 


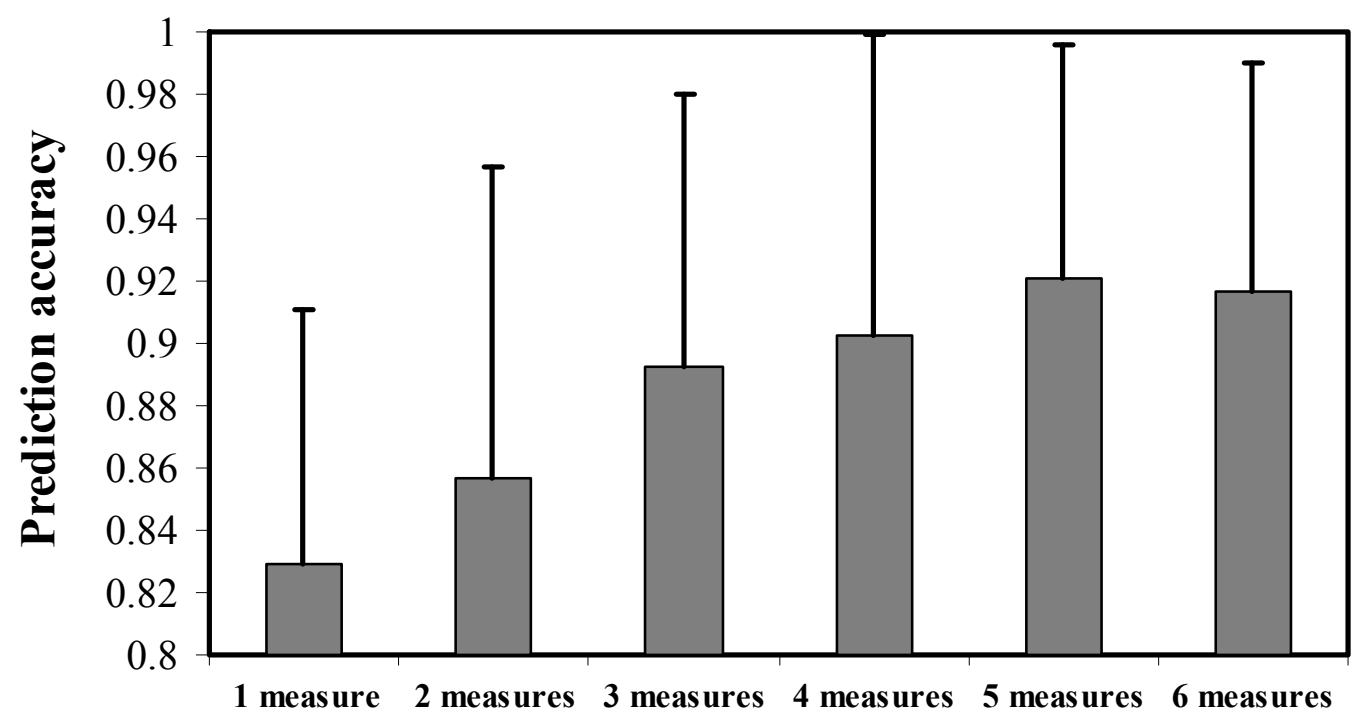

Fig. 10 Comparison of prediction accuracy among the numbers of behavioral measures entered into the multinomial logistic regression model. The independent variables were entered into the model according to the circled number in Fig. 9. The tracking error was entered unconditionally. For two measures, the circled numbers 1 (tracking error) and 2 (back pressure) were entered. For three measures, the circled numbers 1, 2 and 3 (foot pressure) were entered. Alike, for six measures, the circled numbers 1 to 6 were entered.

behavioral measures when only one behavioral measure was entered into the multinomial logistic regression model. Therefore, in both correlation coefficient-based method and the odds ratio-based method, the tracking error was firstly entered into the prediction model. As the foot pressure and the back pressure showed higher correlation coefficients than their difference values, the differences of foot and back pressures were excluded from entering into the prediction model.

These two methods determine the order of entering other five behavioral measures other than the tracking error into the model. No significant difference of prediction accuracy was detected between two determination methods as a result of a statistical $t$-test conducted on the prediction accuracy in Figs. 8 and 10. This means that both methods for determining the order of entering behavioral measures into the prediction model are equally acceptable.

\subsection{Number of Behavioral Measures Used for Prediction}

Multiple comparisons by Fisher's PLSD (protected least significant difference) conducted on the prediction accuracy revealed no significant differences of prediction accuracy among four, five and six behavioral measures in both Figs. 8 and 10. Models with not less than four behavioral measures did not affect the prediction accuracy at all. This indicates that four behavioral measures are enough to achieve higher prediction accuracy. This must be desirable because we can put such a prediction system with fewer behavioral measures and thus leading to less expensive realization of such a system (it is apparent that the system with four measurements is less expensive that that with six).

\subsection{Comparison of Prediction Accuracy between Strongly Drowsy Group and Weakly Drowsy Group}

Although all participants were required to stay up all night, the extent of drowsiness was different across the participants. Such a difference was expressed using the following variable $Z$. The total number of subjective ratings on drowsiness and the number of answer 3 (very drowsy) or no reaction are denoted by $A$ and $B$, respectively. $Z$ is given by the following 
equation:

$$
Z=B / A
$$

As the mean value of $S D$ (standard deviation) for all participants was 0.548 , the participants were divided into the strongly drowsy group and the weakly drowsy group using this mean as a boundary value. Seven and six participants were included in the strongly drowsy group and the weakly drowsy group, respectively.

The mean values of $Z$ for the strongly drowsy group and the weakly drowsy group were 0.738 (SD: 0.111) and 0.328 (SD: 0.105), respectively. As a result of statistical $t$-test, significant difference of $Z$ value was detected between two groups $(|t|=6.78666, p<0.01)$. The prediction accuracy was found to be higher when the participants strongly felt drowsy than when they did not. This suggests that the proposed method is effective especially when the drowsiness is induced to a larger extent.

The prediction accuracy is plotted as a function of the number of behavioral measures entered and the extent of drowsiness in Fig. 11. A two-way (the number of behavioral measures entered by the extent of drowsiness) ANOVA (analysis of variance) conducted on the prediction accuracy revealed significant main effects of the number of behavioral measures entered $(F(5,55)=97.113, p<0.01)$ and the extent of drowsiness $(F(1,11)=4.367, p<0.05)$. Even from Fig. 11, no significant differences of prediction accuracy were detected among four, five and six behavioral measures. This further verified the finding mentioned in Section 4.2 (four behavioral measures are enough for achieving high prediction accuracy).

\subsection{General Discussion}

The practicability of this study can be stated as follows. We can expect that the physiological symptom of drowsiness follows the psychological symptoms of drowsiness and the risk of drowsy driving is high, if the emergence of psychological symptom of drowsiness was successfully detected. In other words, the detection of psychological symptoms enables us to take some countermeasures such as warn drivers of risky state before more serious physiological drowsiness appears. Thus, the accurate prediction technique of subjective rating on drowsiness is essential for taking some countermeasures to assure safety in driving. The predicted subjective rating on drowsiness can be practically used as follows. If the rating score of 3 (very drowsy) was detected $x$ times in a row, or $y$ times out of $z$, we must force drivers to stop driving somehow. The parameters $x, y$ and $z$ must be determined empirically.

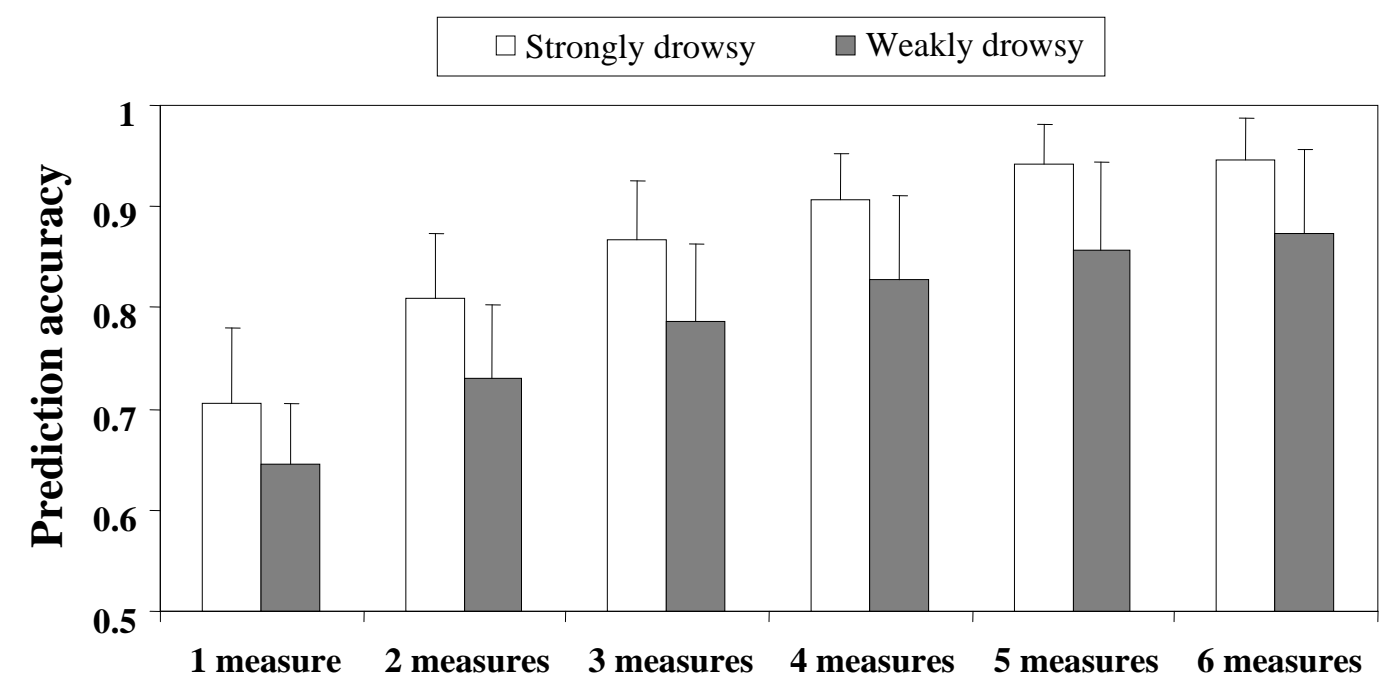

Fig. 11 Prediction accuracy compared among the numbers of behavioral measures entered into the model and between strongly drowsy group and weakly drowsy group. 
It is further necessary to identify the time when the driver is sure to fall asleep and, at worst case, bring about a crucial traffic accident and explore whether it is possible to predict such timing in advance using the behavioral measures adopted in this study.

\section{Conclusions}

We showed that the multinomial logistic regression model can predict the subjective rating (evaluation) on drowsiness with high accuracy. From a practical viewpoint, it is desirable to obtain high accuracy with fewer evaluation measures. We proposed a method that assures high prediction accuracy and determined the minimum number of evaluation measures that can effectively predict the subjective drowsiness. The conclusions can be summarized as follows:

(1) When predicting the subjective drowsiness using multinomial logistic regression model, the selection of entered variables using correlation coefficient or odds ratio was equally useful;

(2) The minimum number of variables that can be used most effectively for prediction was four in the range of this study;

(3) The prediction accuracy differed between the strongly and the weakly drowsy groups, and the proposed method was found to be effective especially when drowsiness was induced to a larger extent.

After the multinomial logistic regression model was identified using all of the data gathered during the experiment, the prediction of subjective rating on drowsiness at each point in time was conducted (on an off-line basis) by entering the mean behavioral data (just before the subjective drowsiness was reported by the switch response) into the model. Future research should carry out such a prediction on an on-line basis so that a warning can be provided with drivers as soon as the system detected the drowsy and risky state.

\section{Acknowledgments}

This work was partly supported by Grant-in Aid for
Scientific Research (B) (22310101, 26282095), JSPS (Japan Society for the Promotion of Science).

\section{References}

[1] Skipper, J. H., and Wierwillie, W. 1986. "Drowsy Driver Detection Using Discrimination Analysis.” Human Factors 28 (5): 527-40.

[2] Brookhuis, K. A., and Waard, D. 1993. "The Use of Psychophysiology to Assess Driver Status.” Ergonomics 36 (9): 1099-110.

[3] Kecklund, G., and Akersted, T. 1993. "Sleepiness in Long Distance Truck Driving: An Ambulatory EEG Study of Night Driving.” Ergonomics 36 (9) : 1007-17.

[4] Murata, A., and Hiramatsu, Y. 2008. "Evaluation of Drowsiness by HRV Measures-Basic Study for Drowsy Driver Detection.” In Proceedings of IWCIA (International Workshop on Computational Intelligence and Applications), 99-102.

[5] Murata, A., and Nishijima, K. "Evaluation of Drowsiness by EEG Analysis-Basic Study on ITS Development for the Prevention of Drowsy Driving." In Proceeings of IWCIA2008, 95-8.

[6] Murata, A., Matsuda, Y., Moriwaka, M., and Hayami, T. 2011. "An Attempt to Predict Drowsiness by Bayesian Estimation.” In Proceeding of SICE (Society of Instrument and Control Engineers) 2011, 58-63.

[7] Murata, A., Ohkubo, Y., Moriwaka, M., and Hayami, T. 2011. "Prediction of Drowsiness Using Multivariate Analysis of Biological Information and Driving Performance.” In Proceedings of SICE 2011, 52-57.

[8] Murata, A., Koriyama, T., and Hayami, T. 2012. "Basic Study on the Prevention of Drowsy Driving Using the Change of Neck Bending Angle and the Sitting Pressure Distribution.” In Proceedings of SICE 2012, 274-9.

[9] Murata, A., Koriyama, T., Ohkubo, Y., Moriwaka, M., and Hayamai, T. 2013. "Verification of Physiological or Behavioral Evaluation Measures Suitable for Predicting Drivers' Drowsiness.” In Proceedings of SICE 2013, 1766-71.

[10] Murata, A., Nakatsuka, A., and Moriwaka, M. 2013. "Effectiveness of Back and Foot Pressures for Assessing Drowsiness of Drivers.” In Proceedings of SICE 2013, 1754-9.

[11] Murata, A., Urakami, Y., Koriyama, T., Ikeda, M., and Hayami, T. 2013. "Evaluation of Drowsiness of Driver Based on Change of Sitting Pressure Center." In Proceedings of SICE 2013, 1760-5.

[12] Schwartz, B. 2005. The Paradox of Choice: Why More Is Less. New York: Harper Perennial. 